Western University

Scholarship@Western

Bone and Joint Institute

$1-1-2020$

Comparison of Contact Kinematics in Posterior-Stabilized and

Cruciate-Retaining Total Knee Arthroplasty at Long-Term Follow-

Up

Jordan S. Broberg

Western University

Silvio Ndoja

London Health Sciences Centre

Steven J. MacDonald

London Health Sciences Centre

Brent A. Lanting

London Health Sciences Centre

Matthew G. Teeter

Western University

Follow this and additional works at: https://ir.lib.uwo.ca/boneandjointpub

Part of the Medicine and Health Sciences Commons

Citation of this paper:

Broberg, Jordan S.; Ndoja, Silvio; MacDonald, Steven J.; Lanting, Brent A.; and Teeter, Matthew G., "Comparison of Contact Kinematics in Posterior-Stabilized and Cruciate-Retaining Total Knee Arthroplasty at Long-Term Follow-Up" (2020). Bone and Joint Institute. 756.

https://ir.lib.uwo.ca/boneandjointpub/756 
Basic Science

\title{
Comparison of Contact Kinematics in Posterior-Stabilized and Cruciate-Retaining Total Knee Arthroplasty at Long-Term Follow-Up
}

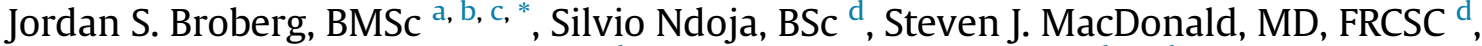 \\ Brent A. Lanting, MD, MSc, FRCSC ${ }^{\mathrm{d}}$, Matthew G. Teeter, PhD ${ }^{\mathrm{a}, \mathrm{b}, \mathrm{c}, \mathrm{d}}$ \\ a Department of Medical Biophysics, Schulich School of Medicine and Dentistry, Western University, London, Ontario, Canada \\ ${ }^{\mathrm{b}}$ Imaging Research Laboratories, Robarts Research Institute, Schulich School of Medicine and Dentistry, Western University, London, Ontario, \\ Canada \\ c Surgical Innovation Program, Lawson Health Research Institute, London, Ontario, Canada \\ ${ }^{\mathrm{d}}$ Division of Orthopaedic Surgery, Department of Surgery, Schulich School of Medicine and Dentistry, Western University and London Health \\ Sciences Centre, London, Ontario, Canada
}

\section{A R T I C L E I N F O}

\section{Article history:}

Received 7 June 2019

Received in revised form

18 July 2019

Accepted 31 July 2019

Available online 6 August 2019

\section{Keywords:}

total knee arthroplasty

posterior-stabilized

cruciate-retaining

kinematics

radiostereometric analysis

patient function

\begin{abstract}
A B S T R A C T
Background: There is controversy regarding the superiority of posterior-stabilizing (PS) total knee arthroplasty (TKA) and cruciate-retaining (CR) TKA. Substantial work has made comparisons between PS and CR TKA at follow-ups of less than 5 years. It was the goal of the present study to compare the kinematics at greater than 5 years postoperatively between CR and PS TKA, with a secondary goal of comparing patient function.

Methods: A total of 42 knees were investigated, with equal representation in the PS and CR TKA groups. Patients underwent radiostereometric analysis imaging at $0^{\circ}, 20^{\circ}, 40^{\circ}, 60^{\circ} 80^{\circ}$, and $100^{\circ}$ of flexion Contact position, magnitude of excursion, and condylar separation on each condyle were measured. A Timed-Up-and-Go functional test was also performed by patients, with the total test time being measured. Preoperative and postoperative clinical outcome scores were also collected.

Results: There were differences in contact position on both the medial and lateral condyles at multiple angles of flexion $(P<.05)$. There was no difference $(P=.89)$ in medial excursion; however, PS TKA had greater lateral excursion than CR TKA $(P<.01)$. No difference $(P>.99)$ was found in frequency of condylar separation. PS TKA was associated with faster $(P=.03)$ total Timed-Up-and-Go test times. There were no differences in clinical outcome scores between the groups preoperatively or postoperatively.

Conclusion: We found kinematic and functional differences that favor PS TKA. Our results suggest posterior cruciate ligament insufficiency in CR TKA, indicating that perhaps the cam/post systems in PS TKA better maintain knee kinematics and function long term.
\end{abstract}

() 2019 Elsevier Inc. All rights reserved.
The decision to retain or sacrifice the posterior cruciate ligament (PCL) in total knee arthroplasty (TKA) has been a longstanding debate within the field of orthopedics. Knee arthroplasties that

\footnotetext{
One or more of the authors of this paper have disclosed potential or pertinent conflicts of interest, which may include receipt of payment, either direct or indirect, institutional support, or association with an entity in the biomedical field which may be perceived to have potential conflict of interest with this work. For full disclosure statements refer to https://doi.org/10.1016/j.arth.2019.07.046.

* Reprint requests: Jordan S. Broberg, BMSc, Department of Medical Biophysics, Schulich School of Medicine and Dentistry, Western University, 1151 Richmond Street, London, ON N6A 3K7, Canada.
}

retain the PCL and those that sacrifice the PCL have both existed since the explosion of innovation in TKA prosthetic designs that occurred in the 1970s [1]. Advocates of cruciate-retaining (CR) designs argue that retaining the PCL provides more natural knee kinematics, improved proprioception, and enhanced inherent stability [2,3]. Those in favor of posterior-stabilized (PS) TKA, where the PCL is sacrificed and replaced by a cam/post system, claim that the PS design allows for better ligament balancing, a simpler surgical procedure, reduced tibiofemoral loads, and more predictable kinematics [4]. Comparisons between PS and CR TKA have been made in multiple meta-analyses, where a broad range of results were examined, including clinical and radiological outcomes, 
flexion and extension angles, range of motion, survivorship, and complication rates [5-8]. The consensus from these findings suggests that while PS TKA allows for greater range of motion and flexion angles, there are no clinically relevant differences that would indicate the use of one design over the other.

Another important factor to consider when comparing TKA designs is the contact kinematics. Common deviations from normal kinematics in replaced knees, such as reduced posterior femoral rollback, paradoxical anterior femoral translation, and femoral condylar separation, can lead to multiple issues [9,10]. Paradoxical anterior femoral translation causes an anterior positioning of the flexion axis that decreases maximum flexion, results in a decreased quadriceps moment arm that reduces quadriceps efficiency, and has been shown to increase the risk of accelerated polyethylene wear [10-12]. Greater posterior femoral rollback has been associated with increased flexion, because more rollback delays posterior impingement between the femur and tibia [9,10]. Furthermore, kinematic abnormalities have been correlated with an increase in tibial component migration, which can lead to early loosening and implant failure [13]. Therefore, it should be the goal of TKA designs to limit the occurrence of these abnormalities.

While substantial work has been performed investigating the kinematics of PS and CR designs at short-term follow-ups of less than 5 years, to our knowledge, there has not been a long-term examination of the kinematics in PS and CR TKA. A study of the kinematics at long-term follow-up is important to further assess whether the kinematics of each design are maintained. Thus, it is the goal of the present study to compare the kinematics at greater than 5 years postoperatively between PS and CR TKA. An examination of patient function was also completed to determine whether kinematic differences led to functional disparities between groups.

\section{Materials and Methods}

Forty-five patients (50 knees) were recruited for the study, and patients were matched according to their sex, age within 2 years, body mass index within $3 \mathrm{~kg} / \mathrm{m}^{2}$, and date of surgery within 6 months. Included in the matching protocol were patients who were at least 5 years postoperation from a TKA where either a PS version or a $\mathrm{CR}$ version of the same prosthesis was used (Sigma; DePuy, Warsaw, IN). Exclusion criteria included patients who did not receive the implant studied, those who did not have their surgery performed at University Hospital, London Health Sciences Center, and those who had a follow-up less than 5 years.

Of the 50 knees recruited, 4 matched pairs were excluded because of unmeasurable images in the kinematic analysis. There was equal representation of the remaining 42 knees recruited in both the PS and the CR groups, resulting in 21 knees per group. Patient demographics are displayed in Table 1 . There were no significant differences in age $(P=.34)$, sex $(P>.99)$, body mass index $(P=.79)$, or years postoperation $(P=.15)$ between the PS and the CR groups. Average follow-up exceeded 9 years in each group.

Patients in both groups had previously received a cobaltchromium on conventional polyethylene TKA with a resurfaced patella (Sigma; DePuy) using cemented fixation. The PS group

Table 1

Patient Demographics, Presented as Mean \pm Standard Deviation.

\begin{tabular}{lllc}
\hline Variable & Posterior-Stabilized & Cruciate-Retaining & $P$ Value \\
\hline Age $(\mathrm{y})$ & $65.6 \pm 5.07$ & $66.1 \pm 5.60$ & .34 \\
Body mass index $\left(\mathrm{kg} / \mathrm{m}^{2}\right)$ & $32.7 \pm 5.23$ & $32.9 \pm 4.97$ & .79 \\
Sex & 9 male, 12 female & 9 male, 12 female & $>.99$ \\
Years postoperation & $9.49 \pm 1.84$ & $9.36 \pm 1.79$ & .15 \\
\hline
\end{tabular}

consisted of patients who had received a PS Sigma prosthesis from a single high-volume surgeon who preferred PS designs, whereas the $\mathrm{CR}$ group was comprised of patients who had received a CR Sigma prosthesis from a different high-volume surgeon who favored CR designs. Other than the version of the prosthesis used, all aspects of the operation and postoperative care and rehabilitation were identical between the 2 groups and occurred within the same hospital and physiotherapy department. Both surgeons used a measured resection surgical technique. In the $\mathrm{CR}$ group, the $\mathrm{PCL}$ was partially released if the knee was too tight in flexion.

Patients recruited to the study underwent weight-bearing stereo X-ray examinations using a radiostereometric analysis (RSA) system at multiple angles of flexion $\left(0^{\circ}, 20^{\circ}, 40^{\circ}, 60^{\circ}, 80^{\circ}\right.$, and $\left.100^{\circ}\right)$ [14]. A uniplanar calibration cage (RSA Biomedical, Umea, Sweden) was used for every examination. The 2D/3D registration of the manufacturer's CAD models for the femoral and tibial implant components to each stereo X-ray pair was performed using modelbased RSA software (RSAcore, Leiden, Netherlands), which has been demonstrated to have measurement errors of $0.19 \mathrm{~mm}$ for translations and $0.52^{\circ}$ for rotations [15]. After obtaining the positions and orientations for both the registered femoral and tibial components, a model of the polyethylene liner with the correct thickness was added to the tibial baseplate model. An in-house software program was used to find the point of shortest distance between the femoral and tibial components on each of the medial and lateral condyles, as well as the magnitude of the distance between components. A difference of $0.5 \mathrm{~mm}$ between the tibiofemoral distances of the medial and lateral condyles was chosen as the threshold to be considered condylar separation, matching a previous study where RSA imaging was used to acquire kinematic data [16].

In addition to the kinematic data, the functional ability of patients was determined using a standardized walking test known as the Timed-Up-and-Go (TUG) test [17-19]. During this test, the patient stands up from a chair, walks $3 \mathrm{~m}$ to a measured goal, turns around at the goal, walks back to the chair, and sits down in the chair [19]. The total time to complete the TUG test was measured, with shorter times indicative of patients with higher function.

Clinical outcome scores from both the preoperative time point and the postoperative time point at which the patient visited for the study were recorded. Included scores were the Short Form 12 (SF-12) mental component score (MCS) and physical component score (PCS), the Knee Society Score (KSS), and the Western Ontario and McMaster Universities Osteoarthritis Index (WOMAC).

All statistical analyses were performed using Prism 8. Normality of all the data was tested using a D'Agostino and Pearson omnibus normality test. Comparisons between the group demographics were done using a paired $t$-test for normally distributed data, or a Wilcoxon matched-pairs signed-rank test when the data were not normally distributed. The ratios of male to female were determined using a Fisher exact test. Comparisons between groups for the clinical outcome scores were done using a paired $t$-test for normally distributed data, or a Wilcoxon matched-pairs signed-rank test when the data were not normally distributed. Comparisons between the preoperative and postoperative clinical outcome scores within each group were performed with a paired $t$-test when data were normally distributed, or a Wilcoxon matched-pairs signedrank test when the data were not. A Fisher exact test was used to compare the frequency of condylar separation between groups. For each degree of flexion analyzed, the mean anteroposterior (AP) contact position on both the medial and lateral condyle was determined. A paired $t$-test or Wilcoxon matched-pairs signed-rank test was done depending on whether the data were normal or not, respectively, to compare the AP position of each group for both the medial and lateral condyle. Excursion (the range between minimum and maximum AP contact position) was calculated across all 
A Posterior-Stabilized

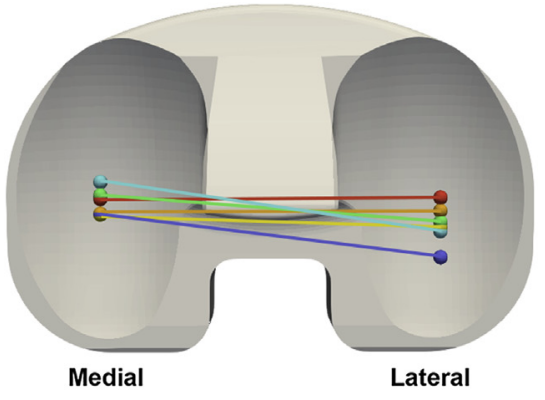

B Cruciate-Retaining

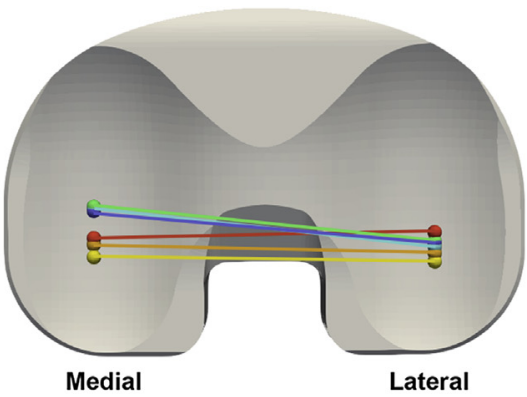

Flexion: $0^{\circ} \quad 20^{\circ} \quad 40^{\circ} \quad 60^{\circ} 80^{\circ} 100^{\circ}$

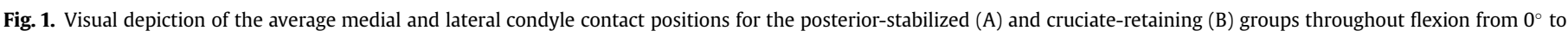
$100^{\circ}$.

degrees of flexion, and comparisons between groups were made using a paired $t$-test for normally distributed data or a Wilcoxon matched-pairs signed-rank test for non-normal data. Comparing the total TUG test time between the groups was done using a MannWhitney test. The statistical significance threshold was set at $P=.05$.

\section{Results}

On the medial condyle, the PS group (Fig. 1A) consistently exhibited more anterior contact than the CR group (Fig. 1B) throughout early flexion at $0^{\circ}(P=.004), 20^{\circ}(P=.045)$, and $40^{\circ}(P=$ $.002)$, where the average magnitude of this difference ranged from $3.5 \mathrm{~mm}$ at $20^{\circ}$ to $5.1 \mathrm{~mm}$ at $40^{\circ}$ (Fig. 2A). The PS and CR groups showed similar contact positions at $60^{\circ}(P=.94)$ before the PS group demonstrated more anterior contact at $80^{\circ}(P=.047)$ of flexion, where the average magnitude of difference was $3.2 \mathrm{~mm}$. The PS and CR groups displayed similar contact positions again at $100^{\circ}(P=.54)$ of flexion. In both groups, the medial contact position moved posteriorly from $0^{\circ}$ to $40^{\circ}$, then moved anteriorly from $40^{\circ}$ to $60^{\circ}$. In the PS group, the medial contact position continued to shift anteriorly from $60^{\circ}$ to $80^{\circ}$ before moving posteriorly from $80^{\circ}$ to $100^{\circ}$. In the $\mathrm{CR}$ group, the contact position moved slightly posteriorly from $60^{\circ}$ to $80^{\circ}$.

A similar trend was seen on the lateral condyle (Fig. 2B), where the PS group had consistently more anterior contact positions than the $\mathrm{CR}$ group throughout early flexion at $0^{\circ}(P=.02), 20^{\circ}(P=.03)$, and $40^{\circ}(P=.0003)$, and the average magnitude of this difference ranged from $4.0 \mathrm{~mm}$ at $0^{\circ}$ to $5.0 \mathrm{~mm}$ at $20^{\circ}$. The PS and CR groups showed similar contact positions from $60^{\circ}(P=.13)$ to $80^{\circ}(P=.10)$ of flexion before the PS group demonstrated posterior contact relative to the $\mathrm{CR}$ group at $100^{\circ}(P=.02)$ of flexion, where the average magnitude of difference was $2.7 \mathrm{~mm}$. In both groups, the lateral contact position moved posteriorly from $0^{\circ}$ to $40^{\circ}$. The lateral contact position for the PS group remained relatively constant, moving only slightly anteriorly from $40^{\circ}$ to $60^{\circ}$ before shifting back to a slightly posterior position from $60^{\circ}$ to $80^{\circ}$. The lateral contact position for the CR group had a constant anterior shift from $40^{\circ}$ to $60^{\circ}$ before a slight posterior shift from $60^{\circ}$ to $80^{\circ}$. The PS group displayed a posterior shift in lateral contact from $80^{\circ}$ to $100^{\circ}$, whereas the lateral contact position for the CR group shifted slightly anteriorly from $80^{\circ}$ to $100^{\circ}$.

There was no significant difference $(P=.89)$ in the average medial excursion between the PS group $(8.9 \pm 4.4 \mathrm{~mm})$ and the CR group $(9.1 \pm 2.7 \mathrm{~mm})$. However, there was a difference $(P=.0016)$ in the average lateral excursion, with the PS group $(10.1 \pm 6.0 \mathrm{~mm})$ displaying a greater average lateral excursion than the CR group $(5.9 \pm 2.8 \mathrm{~mm})$.
No difference $(P>.99)$ was found in the frequency of condylar separation between the PS group ( $n=6$ of 21 patients) and the CR group ( $n=5$ of 21 patients). In the PS group, 1 of the 7 instances was medial separation and 5 of the 6 were lateral separation. In the CR group, 3 of the 5 instances were medial separation and 2 of the 5 were lateral separation.

There was a significant difference $(P=.03)$ in total TUG test time between the PS group $(12.4 \pm 3.4 \mathrm{~s}, \mathrm{n}=11)$ and the $\mathrm{CR}$ group $(14.9 \pm$ $3.3 \mathrm{~s}, \mathrm{n}=11$ ).

There were no significant differences present in the SF-12, KSS, or WOMAC outcome scores between the PS and CR groups preoperatively or postoperatively (Table 2). The PS group showed significant improvements preoperatively to postoperatively in the SF-12 PCS ( $P$ $<.0001)$, KSS $(P<.0001)$, and WOMAC $(P<.0001)$ outcome scores; however, no difference in the SF-12 MCS $(P=.38)$. Likewise, the CR group showed improvements preoperatively to postoperatively in the SF-12 PCS $(P=.0001)$, KSS $(P<.0001)$, and WOMAC $(P<.0001)$ outcome scores, and no difference in the SF-12 MCS $(P=.62)$.

\section{Discussion}

The purpose of TKA is to provide the patient with a stable knee that functions well to provide good satisfaction for a long period of time. However, while TKA survivorship is good, patient satisfaction with their arthroplasty could be improved [20-22]. Understanding how the kinematics of the knee are affected by the design of the knee arthroplasty is important in the goals of a TKA-a satisfied patient with good implant longevity. Many investigations have focused specifically on the knee kinematics of CR and PS designs. Dennis et al [9] conducted a large multicenter study looking at the kinematics of various CR and PS designs during gait and deep knee bends at a minimum of 1 year postoperatively. Similar kinematic patterns were seen during gait, where the PCL and cam/post function are minimal. However, during a deep knee bend, the PS designs consistently showed greater posterior femoral rollback, which allows for increased knee flexion. Another study also found that at an average of 2 years postoperatively, a high flexion PS design allowed for greater weight-bearing maximal flexion and posterior femoral rollback when compared to a high flexion CR TKA [23]. A study looking at condylar liftoff at a minimum of 1 year postoperatively found that both CR and PS designs experienced similarly high rates of condylar liftoff, mostly at the greater flexion angles [24]. One study investigating the kinematics of CR and PS designs during a stair climb at an average of 2-3 years postoperatively found that the CR design provided mid-flexion stability, as anterior translation of the femur was absent, whereas in the PS design anterior translation was present, indicating it did not 

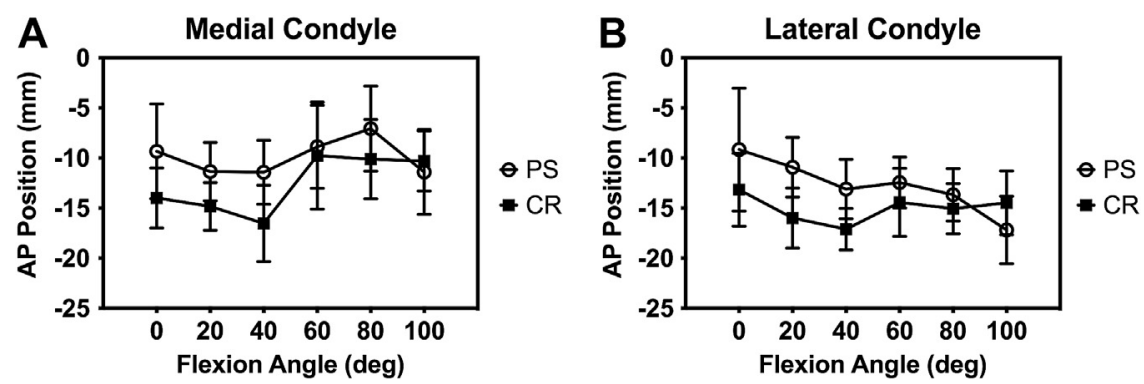

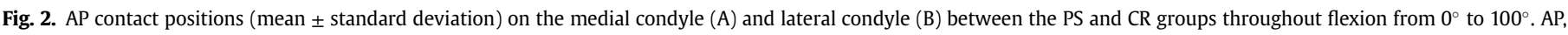
anteroposterior; PS, posterior-stabilized; CR, cruciate-retaining.

provide mid-flexion stability during a stair climb [25]. Another study with a follow-up of 5 years postoperatively demonstrated that during both a deep lunge activity and a stair climb activity, the PS design had greater and more consistent posterior femoral rollback and less paradoxical anterior translation [26]. A recent review article on TKA kinematics corroborated many of these findings, reporting that $\mathrm{CR}$ designs experienced a greater frequency of anterior translation and less posterior femoral rollback than PS designs during a weight-bearing deep knee bend [10].

While substantial work has been performed investigating CR and PS designs at short-term follow-ups of less than 5 years, few studies have reported long-term findings comparing CR and PS designs. One long-term study demonstrated no clinically relevant differences in implant longevity, pain relief, or functional improvement up to 10 years postoperatively [27]. Another longterm study found that while survivorship of the 2 designs was similar, the PS design was associated with greater range of motion and better clinical outcome scores at 10 years postoperatively [28]. Recently, a study examining the Australian Orthopaedic Association National Joint Replacement Registry has shown better long-term survivorship for CR designs [20]. However, to our knowledge, there has been no previous long-term examination of the kinematics in PS and CR TKA. With prosthetics lasting well into their second decade, both PS and CR designs should aim to limit the occurrence of kinematic abnormalities not only in the short-term but also in the long-term. Many studies have shown that PS designs typically outperform CR designs with respect to contact kinematics, and that both are unable to replicate normal knee kinematics at early follow-ups $[9,10,26]$. Our findings show that this remains true nearly a decade postoperation.

PS TKA had a significantly more anterior tibiofemoral contact position for both medial and lateral condyles than CR TKA at early flexion, perhaps due to the lack of a PCL that would prevent an

Table 2

Patient Outcome Scores at Preoperative and Postoperative Time Points, Presented as Mean \pm Standard Deviation.

\begin{tabular}{cccc}
\hline Variable & Posterior-Stabilizing & Cruciate-Retaining & $P$ Value \\
\hline SF-12 MCS & & & \\
Preop & $57.0 \pm 8.9$ & $56.5 \pm 9.5$ & .88 \\
Postop & $53.0 \pm 9.8$ & $53.8 \pm 8.9$ & .82 \\
SF-12 PCS & & & \\
Preop & $29.4 \pm 6.4$ & $28.3 \pm 6.6$ & .52 \\
Postop & $42.2 \pm 11.5$ & $40.2 \pm 12.1$ & .62 \\
KSS & & & .74 \\
Preop & $100.1 \pm 16.8$ & $98.1 \pm 23.4$ & .06 \\
Postop & $183.6 \pm 26.4$ & $176.2 \pm 29.9$ & .65 \\
WOMAC & & & $>.99$ \\
Preop & $46.9 \pm 17.6$ & $50.0 \pm 18.5$ & \\
Postop & $79.5 \pm 20.7$ & $79.8 \pm 23.1$ & \\
\hline
\end{tabular}

$\overline{\text { SF-12, Short Form 12; MCS, mental component score; PCS, physical component }}$ score; KSS, Knee Society Score; WOMAC, Western Ontario and McMaster Universities Osteoarthritis Index; preop, preoperative; postop, postoperative. anterior positioning of the femur. However, at $60^{\circ}$ of flexion, both designs have a similar contact point on both condyles. While the medial contact position of the PS group is more anterior at $80^{\circ}$, the CR design still experiences greater paradoxical anterior translation of the femur than the PS design. While paradoxical anterior femoral translation was still present in the PS TKA group, it was less evident.

Additionally, there is a clear difference in the amount of posterior femoral rollback between the 2 designs. One report on normal knee kinematics shows that the normal knee will experience an average of $6.9 \mathrm{~mm}$ and $27.4 \mathrm{~mm}$ of posterior femoral rollback during a deep knee bend from $0^{\circ}$ to $120^{\circ}$ on the medial and lateral condyles, respectively, indicating the presence of a medial pivot kinematic pattern [29]. Another study showed similar findings for normal knees, with averages of $1.9 \mathrm{~mm}$ and $21 \mathrm{~mm}$ of posterior femoral rollback medially and laterally, respectively [30]. We can infer the amount of posterior femoral rollback in both PS and CR TKA in our study by analyzing the excursions. Our study found that PS TKA had a medial and lateral excursion of 8.9 and $10.1 \mathrm{~mm}$, respectively, whereas CR TKA had a medial excursion of $9.1 \mathrm{~mm}$ and a lateral excursion of $5.9 \mathrm{~mm}$. In both groups, there is a noticeable increase in posterior translation medially and a reduction laterally when compared to the normal knee.

In agreement with a report by Dennis et al [24] that used the same implant, the frequency of condylar separation was similar between CR and PS TKA; however, our results show a lower overall frequency of separation than what was found in their study. While Dennis et al studied condylar liftoff, which uses the femoral component and the tibial tray to determine the tibiofemoral distances, our study analyzed condylar separation, which uses the polyethylene insert in place of the tibial tray when determining the tibiofemoral distance [31-33]. It is not likely that this caused the discrepancy in the frequency of liftoff/separation because the implant has symmetrical medial and lateral condyles. Another major difference in our findings from the Dennis et al's study was the frequency of medial and lateral liftoff in both groups. Our results indicate that condylar separation is predominately lateral in PS TKA and occurs slightly more often medially in CR TKA. Dennis et al [24] observed more lateral liftoff in the CR TKA group, suggesting that the PCL functions to prevent medial liftoff due to its attachment to the medial femoral condyle; however, these results were from a minimum follow-up of 1 year. Our results suggest that perhaps the PCL has a decreased ability to prevent medial liftoff in CR TKA over a longer time of follow-up.

Furthermore, the medial pivot kinematic pattern present in normal knees was absent in both groups [9]. In the PS TKA group, no prominent medial or lateral pivot pattern was seen, while in the $C R$ TKA group a lateral pivot pattern was evident, a drastic change from the normal medial pivot pattern. A possible explanation for this is the increased medial condylar separation in CR TKA. With less medial contact in the CR designs, the medial condyle is unable to act as the pivot point. Instead the lateral condyle acts as the pivot point 
and external rotation of the femur occurs through anterior translation of the medial condyle rather than through the posterior rollback of the lateral condyle, a common phenomenon in CR TKA [9,26,34].

Despite the difference in kinematics between the PS and CR groups, no differences were observed in multiple patient questionnaires, supporting previous results from Beaupre et al [27], who compared PS and CR TKA at 10 years postoperatively. However, these measures can be influenced by many other factors and therefore are not always reliable [35]. When using the total TUG test time, an objective measurement of patient function, we found a clinically relevant difference greater than the TUG test minimally clinically important difference of 2.27 seconds in patient function, with the PS group outperforming the CR group [17]. One study that used the TUG test to analyze the fall risk of patients found that the TUG test time of nonfallers was 12.4 seconds and was 15.6 seconds for fallers [36]. Our results suggest that patients in the PS group have total TUG test times similar to that of nonfallers and that patients in the CR group have times more closely resembling that of fallers. Although multiple factors may be causal to this difference in times, one potential reason could be the more kinematic abnormalities associated with CR TKA.

A healthy knee relies on the PCL to prevent the anterior sliding of the femur and induce posterior femoral rollback, especially laterally, at the higher angles of flexion [37,38]. CR designs also rely on an intact and functional PCL to maintain the knee kinematics. While it has been reported that the PCL remains functional at a follow-up of 4 years, many other histologic reports have shown that the PCL is often already deficient in osteoarthritic knees before surgery [39-41]. In their study of PS vs CR designs 10 years postoperatively, Sando et al [28] suggested that the degeneration of the retained PCL may result in kinematic abnormalities in CR TKA at long-term follow-up. Our results suggest that this may be the case.

This study was not without its limitations. All images were collected with patients in a weight-bearing position, but they were collected using a quasistatic method as opposed to a continuous dynamic deep knee bend. However, multiple studies have shown that static and dynamic kinematics are comparable following TKA, and our findings were consistent with many of the fluoroscopic studies $[9,26,34,42,43]$. Another limitation was the use of the CR and PS versions of only one implant design. The report from Dennis et al [9] showed that even different models of the same type of implant can have different knee kinematics; however, it was important to limit the present study to one particular implant to reduce the influence of different implant model designs and focus on the effects of retaining or sacrificing the PCL. More long-term follow-up studies of different implant models are required to make more general conclusions. Finally, because this was an expertise-based study, surgeon variability may have influenced the results.

In conclusion, we found kinematic differences at an average of 9 years postoperatively that favor fixed-bearing PS knee arthroplasties over their CR counterparts. The PS group displayed less paradoxical anterior femoral translation on both the medial and lateral condyles, and greater lateral condyle excursion indicating greater posterior femoral rollback. No differences between groups were observed in the medial excursion or frequency of condylar separation. Furthermore, there was no difference between groups for the clinical outcome scores, which improved from preoperatively to the most recent postoperative follow-up. However, there was a difference in total TUG test time, with the PS group demonstrating greater function and a potentially lower fall risk. The increased paradoxical motion, reduced rollback, and lateral pivot in the $\mathrm{CR}$ group may suggest PCL insufficiency in CR TKA, indicating that perhaps the cam/ post systems present in PS TKA allow those designs to better maintain knee kinematics and function in the long term.

\section{Acknowledgments}

This study was funded by the Arthritis Society and an Ontario Early Researcher Award. J.S.B. is supported by an CIHR CGS-M Award. M.G.T. is supported by a CIHR New Investigator Award.

\section{References}

[1] Robinson RP. The early innovators of today's resurfacing condylar knees. J Arthroplasty 2005;20:2-26. https://doi.org/10.1016/j.arth.2004.11.002.

[2] Chalidis BE, Sachinis NP, Papadopoulos P, Petsatodis E, Christodoulou AG Petsatodis G. Long-term results of posterior-cruciate-retaining Genesis I total knee arthroplasty. J Orthop Sci 2011;16:726-31. https://doi.org/10.1007/ s00776-011-0152-1.

[3] In Y, Kim JM, Woo YK, Choi NY, Sohn JM, Koh HS. Factors affecting flexion gap tightness in cruciate-retaining total knee arthroplasty. J Arthroplasty 2009;24: 317-21. https://doi.org/10.1016/j.arth.2007.10.022.

[4] Insall JN, Lachiewicz PF, Burstein AH. The posterior stabilized condylar prosthesis: a modification of the total condylar design. Two to four-year clinical experience. J Bone Joint Surg Am 1982;64:1317-23.

[5] Bercik MJ, Joshi A, Parvizi J. Posterior cruciate-retaining versus posteriorstabilized total knee arthroplasty: a meta-analysis. J Arthroplasty 2013;28: 439-44. https://doi.org/10.1016/j.arth.2012.08.008.

[6] Verra WC, van den Boom LG, Jacobs WC, Schoones JW, Wymenga AB, Nelissen RG. Similar outcome after retention or sacrifice of the posterior cruciate ligament in total knee arthroplasty: a systematic review and meta-analysis. Acta Orthop 2015;86:195-201. https://doi.org/10.3109/17453674.2014.973329.

[7] Li N, Tan Y, Deng Y, Chen L. Posterior cruciate-retaining versus posterior stabilized total knee arthroplasty: a meta-analysis of randomized controlled trials. Knee Surg Sports Traumatol Arthrosc 2014;22:556-64. https://doi.org/ 10.1007/s00167-012-2275-0.

[8] Luo S, Zhao JM, Su W, Li X, Dong G. Posterior cruciate substituting versus posterior cruciate retaining total knee arthroplasty prostheses: a meta-analysis. Knee 2012;19:246-52. https://doi.org/10.1016/j.knee.2011.12.005.

[9] Dennis DA, Komistek RD, Mahfouz MR, Haas BD, Stiehl JB. Multicenter determination of in vivo kinematics after total knee arthroplasty. Clin Orthop Relat Res 2003;416:37-57. https://doi.org/10.1097/01.blo.0000092986.12414.b5.

[10] Angerame MR, Holst DC, Jennings JM, Komistek RD, Dennis DA. Total knee arthroplasty kinematics. J Arthroplasty 2019. https://doi.org/10.1016/ j.arth.2019.05.037. [Epub ahead of print].

[11] Dennis DA, Komistek RD, Hoff WA, Gabriel SM. In vivo knee kinematics derived using an inverse perspective technique. Clin Orthop Relat Res 1996;331:107-17.

[12] Blunn GW, Walker PS, Joshi A, Hardinge K. The dominance of cyclic sliding in producing wear in total knee replacements. Clin Orthop Relat Res 1991;273: 253-60.

[13] Teeter MG, Perry KI, Yuan X, Howard JL, Lanting BA. Contact kinematics correlates to tibial component migration following single radius posterior stabilized knee replacement. J Arthroplasty 2018;33:740-5. https://doi.org/ 10.1016/j.arth.2017.09.064.

[14] Morcos MW, Lanting BA, Webster J, Howard JL, Bryant D, Teeter MG. Effect of medial soft tissue releases during posterior-stabilized total knee arthroplasty on contact kinematics and patient-reported outcomes. J Arthroplasty 2019;34:1110-5. https://doi.org/10.1016/j.arth.2019.02.026.

[15] Valstar ER, de Jong FW, Vrooman HA, Rozing PM, Reiber JH. Model-based Roentgen stereophotogrammetry of orthopaedic implants. J Biomech 2001:34:715-22.

[16] Teeter MG, Perry KI, Yuan X, Howard JL, Lanting BA. Contact kinematic differences between gap balanced vs measured resection techniques for single radius posterior-stabilized total knee arthroplasty. J Arthroplasty 2017;32: 1834-8. https://doi.org/10.1016/j.arth.2016.12.054.

[17] Yuksel E, Kalkan S, Cekmece S, Unver B, Karatosun V. Assessing minimal detectable changes and test-retest reliability of the timed up and go test and the 2-minute walk test in patients with total knee arthroplasty. J Arthroplasty 2017;32:426-30. https://doi.org/10.1016/j.arth.2016.07.031.

[18] Freter SH, Fruchter N. Relationship between timed "up and go" and gait time in an elderly orthopaedic rehabilitation population. Clin Rehabil 2000;14 96-101. https://doi.org/10.1191/026921500675545616.

[19] Podsiadlo D, Richardson S. The timed "Up \& Go": a test of basic functional mobility for frail elderly persons. J Am Geriatr Soc 1991;39:142-8.

[20] Vertullo CJ, Lewis PL, Lorimer M, Graves SE. The effect on long-term survivorship of surgeon preference for posterior-stabilized or minimally stabilized total knee replacement: an analysis of 63,416 prostheses from the Australian Orthopaedic Association National Joint Replacement Registry. J Bone Joint Surg Am 2017;99:1129-39. https://doi.org/10.2106/JBJS.16.01083.

[21] Bourne RB, Chesworth BM, Davis AM, Mahomed NN, Charron KDJ, Met D. Patient satisfaction after total knee arthroplasty who is satisfied and who is not? Clin Orthop Relat Res 2010;468:57-63. https://doi.org/10.1007/s11999009-1119-9.

[22] Price AJ, Alvand A, Troelsen A, Katz JN, Hooper G, Gray A, et al. Knee replacement. Lancet 2018;392:1672-82. https://doi.org/10.1016/S0140-673 6(18)32344-4. 
[23] Seon J-K, Park J-K, Shin Y-J, Seo H-Y, Lee K-B, Song E-K. Comparisons of kinematics and range of motion in high-flexion total knee arthroplasty: cruciate retaining vs. substituting designs. Knee Surg Sports Traumatol Arthrosc 2011;19:2016-22. https://doi.org/10.1007/s00167-011-1434-z.

[24] Dennis DA, Komistek RD, Walker SA, Cheal EJ, Stiehl JB. Femoral condylar liftoff in vivo in total knee arthroplasty. J Bone Joint Surg Br 2001;83:33-9.

[25] Hamai S, Okazaki K, Shimoto T, Nakahara H, Higaki H, Iwamoto Y. Continuous sagittal radiological evaluation of stair-climbing in cruciate-retaining and posterior-stabilized total knee arthroplasties using image-matching Techniques. J Arthroplasty 2015;30:864-9. https://doi.org/10.1016/j.arth.2014.12.027.

[26] Victor J, Banks S, Bellemans J. Kinematics of posterior cruciate ligamentretaining and -substituting total knee arthroplasty: a prospective randomised outcome study. J Bone Joint Surg Br 2005;87:646-55. https://doi.org/ 10.1302/0301-620X.87B5.15602.

[27] Beaupre LA, Sharifi B, Johnston DWC. A randomized clinical trial comparing posterior cruciate-stabilizing vs posterior cruciate-retaining prostheses in primary total knee arthroplasty: 10-year follow-up. J Arthroplasty 2017;32: 818-23. https://doi.org/10.1016/j.arth.2016.08.030.

[28] Sando T, McCalden R, Bourne R, MacDonald S, Somerville L. Ten-year results comparing posterior cruciate-retaining versus posterior cruciate-substituting total knee arthroplasty. J Arthroplasty 2015;30:210-5. https://doi.org/ 10.1016/j.arth.2014.09.009.

[29] Asano T, Akagi M, Tanaka K, Tamura J, Nakamura T. In vivo three-dimensional knee kinematics using a biplanar image-matching technique. Clin Orthop Relat Res 2001;388:157-66.

[30] Dennis DA, Mahfouz MR, Komistek RD, Hoff W. In vivo determination of normal and anterior cruciate ligament-deficient knee kinematics. J Biomech 2005;38:241-53. https://doi.org/10.1016/j.jbiomech.2004.02.042.

[31] Stiehl JB, Dennis DA, Komistek RD, Crane HS. In vivo determination of condylar lift-off and screw-home in a mobile-bearing total knee arthroplasty. J Arthroplasty 1999;14:293-9.

[32] Prins AH, Kaptein BL, Banks SA, Stoel BC, Nelissen RG, Valstar ER. Detecting condylar contact loss using single-plane fluoroscopy: a comparison with in vivo force data and in vitro bi-plane data. J Biomech 2014;47:1682-8. https://doi.org/10.1016/j.jbiomech.2014.02.031.
[33] Kanekasu K, Banks SA, Honjo S, Nakata O. Fluoroscopic analysis of knee arthroplasty kinematics during deep flexion kneeling. J Arthroplasty 2004;19: 988-1003.

[34] Dennis DA, Komistek RD, Colwell CE, Ranawat CS, Scott RD, Thornhill TS, et al. In vivo anteroposterior femorotibial translation of total knee arthroplasty: a multicenter analysis. Clin Orthop Relat Res 1998;356:47-57.

[35] Witvrouw E, Victor J, Bellemans J, Rock B, Van Lummel R, Van Der Slikke R, et al. A correlation study of objective functionality and $\{$ WOMAC $\}$ in total knee arthroplasty. Knee Surg Sports Traumatol Arthrosc 2002;10:347-51. https:/ doi.org/10.1007/s00167-002-0302-2.

[36] Greene B, Donovan A, Roman R-O, Cogan L, Scanaill C, Kenny R. Quantitative falls risk assessment using the timed up and go test. IEEE Trans Biomed Eng 2010;57:2918-26. https://doi.org/10.1109/TBME.2010.2083659.

[37] Goldblatt JP, Richmond JC. Anatomy and biomechanics of the knee. Oper Tech Sports Med 2003;11:172-86. https://doi.org/10.1053/otsm .2003.35911.

[38] Sorger JI, Federle D, Kirk PG, Grood E, Cochran J, Levy M. The posterior cruciate ligament in total knee arthroplasty. J Arthroplasty 1997;12:869-79.

[39] Schuster AJ, von Roll AL, Pfluger D, Wyss T. Anteroposterior stability after posterior cruciate-retaining total knee arthroplasty. Knee Surg Sports Traumatol Arthrosc 2011;19:1113-20. https://doi.org/10.1007/s00167-010-1364-1.

[40] Arbuthnot JE, Wainwright O, Stables G, Rathinam M, Rowley DI, McNicholas MJ. Dysfunction of the posterior cruciate ligament in total knee arthroplasty. Knee Surg Sports Traumatol Arthrosc 2011;19:893-8. https:/| doi.org/10.1007/s00167-010-1234-X.

[41] Akisue T, Stulberg BN, Bauer TW, McMahon JT, Wilde AH, Kurosaka M. Histologic evaluation of posterior cruciate ligaments from osteoarthritic knees. Clin Orthop Relat Res 2002:165-73.

[42] Clément J, Hagemeister N, Aissaoui R, de Guise JA. Comparison of quasi-static and dynamic squats: a three-dimensional kinematic, kinetic and electromyographic study of the lower limbs. Gait Posture 2014;40:94-100. https:/| doi.org/10.1016/j.gaitpost.2014.02.016.

[43] Mu S, Moro-Oka T, Johal P, Hamai S, Freeman MA, Banks SA. Comparison of static and dynamic knee kinematics during squatting. Clin Biomech (Bristol, Avon) 2011;26:106-8. https://doi.org/10.1016/j.clinbiomech.20 10.08.006. 